Article

\title{
Exoenzyme Y Contributes to End-Organ Dysfunction Caused by Pseudomonas aeruginosa Pneumonia in Critically Ill Patients: An Exploratory Study
}

\author{
Brant M. Wagener ${ }^{1,2,+}{ }^{\circledR}$, Naseem Anjum ${ }^{1,+}$, Sarah C. Christiaans ${ }^{1}$, Morgan E. Banks ${ }^{1}{ }^{1}$, \\ Jordan C. Parker ${ }^{1}$, Adam T. Threet ${ }^{1}$, Rashidra R. Walker ${ }^{1}$, Kayla D. Isbell ${ }^{1}{ }^{\circledR}$, Stephen A. Moser ${ }^{3}$, \\ Troy Stevens ${ }^{4,5}$, Mikhail F. Alexeyev ${ }^{4,5}$, Jonathon P. Audia ${ }^{4,6}$, Wito Richter ${ }^{4,7}$, Kierra S. Hardy ${ }^{6}$, \\ Lina Abou Saleh ${ }^{4,7}$, Charity Morgan ${ }^{8}$ and Jean-François Pittet ${ }^{1,9,10, *}$ \\ 1 Department of Anesthesiology and Perioperative Medicine, University of Alabama at Birmingham, \\ Birmingham, AL 35294, USA; bwagener@uabmc.edu (B.M.W.); naseemanjum@uabmc.edu (N.A.); \\ sarahc.ucsf@gmail.com (S.C.C.); morganbanks@uabmc.edu (M.E.B.); jordanparker@uabmc.edu (J.C.P.); \\ athreet1@gmail.com (A.T.T.); rashidra.walker@gmail.com (R.R.W.); kaylaisbell@gmail.com (K.D.I.) \\ 2 Center for Free Radical Biology, University of Alabama at Birmingham, Birmingham, AL 35294, USA \\ 3 Department of Pathology, University of Alabama at Birmingham, Birmingham, AL 35294, USA; \\ smoser@uabmc.edu \\ 4 Center for Lung Biology, College of Medicine, University of South Alabama, Mobile, AL 36688, USA; \\ tstevens@southalabama.edu (T.S.); malexeye@southalabama.edu (M.F.A.); jaudia@southalabama.edu (J.P.A.); \\ ricther@southalabama.edu (W.R.); la1621@jagmail.southalabama.edu (L.A.S.) \\ 5 Department of Physiology and Cell Biology, College of Medicine, University of South Alabama, \\ Mobile, AL 36688, USA \\ 6 Department of Microbiology and Immunology, College of Medicine, University of South Alabama, Mobile, \\ AL 36688, USA; ksh1004@jagmail.southalabama.edu \\ 7 Department of Biochemistry and Molecular Biology, College of Medicine, University of South Alabama, \\ Mobile, AL 36688, USA \\ 8 Department of Biostatistics, University of Alabama at Birmingham, Birmingham, AL 35294, USA; \\ cjmorgan@uab.edu \\ 9 Departments of Surgery and Cell, Developmental, and Integrative Biology, University of Alabama at \\ Birmingham, Birmingham, AL 35294, USA \\ 10 Center for Lung Injury and Repair, University of Alabama at Birmingham, Birmingham, AL 35294, USA \\ * Correspondence: jpittet@uabmc.edu; Tel.: +1-205-975-6656 \\ + These authors contributed equally to this manuscript.
}

Received: 21 May 2020; Accepted: 1 June 2020; Published: 4 June 2020

\begin{abstract}
Pseudomonas aeruginosa is an opportunistic pathogen that causes pneumonia in immunocompromised and intensive care unit (ICU) patients. During host infection, P. aeruginosa upregulates the type III secretion system (T3SS), which is used to intoxicate host cells with exoenzyme (Exo) virulence factors. Of the four known Exo virulence factors (U, S, T and Y), ExoU has been shown in prior studies to associate with high mortality rates. Preclinical studies have shown that ExoY is an important edema factor in lung infection caused by $P$. aeruginosa, although its importance in clinical isolates of P. aeruginosa is unknown. We hypothesized that expression of ExoY would be highly prevalent in clinical isolates and would significantly contribute to patient morbidity secondary to $P$. aeruginosa pneumonia. A single-center, prospective observational study was conducted at the University of Alabama at Birmingham Hospital. Mechanically ventilated ICU patients with a bronchoalveolar lavage fluid culture positive for P. aeruginosa were included. Enrolled patients were followed from ICU admission to discharge and clinical P. aeruginosa isolates were genotyped for the presence of exoenzyme genes. Ninety-nine patients were enrolled in the study. ExoY was present in $93 \%$ of $P$. aeruginosa clinical isolates. Moreover, ExoY alone $\left(\right.$ ExoY $\left.^{+} / \mathrm{ExoU}^{-}\right)$was present in $75 \%$ of P. aeruginosa isolates, compared to $2 \%$ ExoU alone $\left(\right.$ ExoY $\left.^{-} / \mathrm{ExoU}^{+}\right)$. We found that bacteria isolated
\end{abstract}


from human samples expressed active ExoY and ExoU, and the presence of ExoY in clinical isolates was associated with end-organ dysfunction. This is the first study we are aware of that demonstrates that ExoY is important in clinical outcomes secondary to nosocomial pneumonia.

Keywords: Type III secretion system; pneumonia; ExoU; tau; amyloid

Key Contribution: This study indicates that the expression of ExoY is highly prevalent in ICU patients diagnosed with $P$. aeruginosa pneumonia and the exoenzyme activity in clinical isolates is detectable in cellular assays. Furthermore, patients with bacterial isolates containing ExoY have significant end-organ dysfunction and ExoY is clinically important in nosocomial pneumonia.

\section{Introduction}

Pseudomonas aeruginosa is a gram-negative, opportunistic pathogen that is ubiquitous in nature and causes severe nosocomial infections in humans [1]. P. aeruginosa is listed by the World Health Organization as a member of the list of Critical Priority 1, highly antibiotic resistant ESKAPE pathogens [2,3]. P. aeruginosa is a frequent cause of bronchopneumonia in critically ill and immunocompromised patients. Furthermore, P. aeruginosa infection causes significant economic burden as pneumonia is a frequent cause of sepsis. P. aeruginosa is also associated with increased mortality in intensive care unit (ICU) patients due to ventilator-associated pneumonia (VAP) [4,5]. Finally; P. aeruginosa is often virulent and exhibits antimicrobial drug resistance [1].

Previous studies have attempted to underscore the bacterium's pathogenicity that leads to lung injury, bacteremia, sepsis and increased end-organ injury and mortality [6,7]. During host infection, $P$. aeruginosa upregulates the Type III secretion system (T3SS) that is used to intoxicate host cells with exoenzyme (Exo) virulence factors. There are four known exoenzymes, named ExoY, ExoU, ExoS and ExoT, which assist P. aeruginosa in evading host defense systems and remodeling cellular lipids. More specifically, each of these enzymes produce a distinct injury in the host [7-10]; for example, ExoS and ExoT interact with Ras and RhoA GTPases, respectively, to modulate the cytoskeleton and endocytosis activities. ExoS and ExoT also cause ADP ribosylation. ExoU is a phospholipase $\mathrm{A}_{2}\left(\mathrm{PLA}_{2}\right)$ that disrupts the integrity of the lipid membrane and ExoY is a promiscuous nucleotidyl cyclase that breaks down microtubules. A hallmark of the exoenzymes is their requirement for host factors to mediate their activation upon injection into a host cell [11].

ExoY is present in $\sim 90 \%$ of $P$. aeruginosa bacteria [12]. It was first described as a soluble adenylyl cyclase, generating cAMP within the cytosolic compartment. More recent studies have demonstrated that this exoenzyme can generate multiple cyclic nucleotides including, to date, cAMP, cGMP, cUMP and cCMP [13-16]. Importantly, ExoY affects the pulmonary endothelium by inducing inter-endothelial cell gap formation, resulting in edema [17-20]. Finally, recent studies indicate that ExoY is involved in the breakdown of peripheral microtubules and subsequent release of tau and amyloid $\beta$. These events lead to decreased endothelial cell barrier integrity and increased end-organ injury in both the pulmonary system and the brain [21-23].

Preclinical studies reveal that ExoU is associated with increased drug resistance and mortality, followed by ExoS and ExoT in a mouse model [24]. Furthermore, clinical studies have also established that lung infection caused by P. aeruginosa strains expressing ExoU are associated with greater severity of disease and higher mortality [25]. Although ExoU has been associated with severe disease and higher mortality rate compared to ExoS and ExoT, the role of ExoY is not well understood. In this study, we sought to understand whether ExoY contributes to end-organ dysfunction and mortality in critically ill patients who developed a pneumonia caused by ExoY-expressing P. aeruginosa. To this end, we collected $P$. aeruginosa clinical isolates from mechanically ventilated patients, genotyped the strains 
for exoenzyme expression, and compared exoenzyme expression with patients' clinical parameters such as end-organ dysfunction, mortality rate and drug resistance.

\section{Results}

\subsection{Patient Recruitment and Demographics}

ExoU has been associated with increased mortality and drug resistance in previous studies [6,7]. To determine whether the presence of ExoY is associated with patient morbidity and mortality, we recruited patients with primary P. aeruginosa pneumonia to determine the prevalence of ExoY and correlate its presence with end-organ dysfunction. Over the 2-year study period, we screened 181 patients with primary P. aeruginosa lung infection (Figure 1). Of the 181 patients screened, 99 met inclusion criteria and were included in the study. Table 1 shows the demographics and characteristics of patients recruited to the study. Of note, the mean age was 57 years, males comprised $68 \%$ of the patients and $49 \%$ were admitted for acute trauma. Sixty-three percent were white, $33 \%$ were black and $6 \%$ were Hispanic. Social habits include that 33\% consume alcohol regularly, 29\% were smokers and $11 \%$ had documented illicit drug use. At ICU admission, the median Acute Physiology and Chronic Health Evaluation (APACHE) II score, Injury Severity Score (ISS), Sepsis-related Organ Failure Assessment (SOFA) and Organ Dysfunction and Infection System (ODIN) score were 16, 18, 7 and 1, respectively (Table 2). Patients were in the ICU for a median of 24 days, were mechanically ventilated for a median of 21 days and were mechanically ventilated for a median of 8 days before the onset of P. aeruginosa infection.

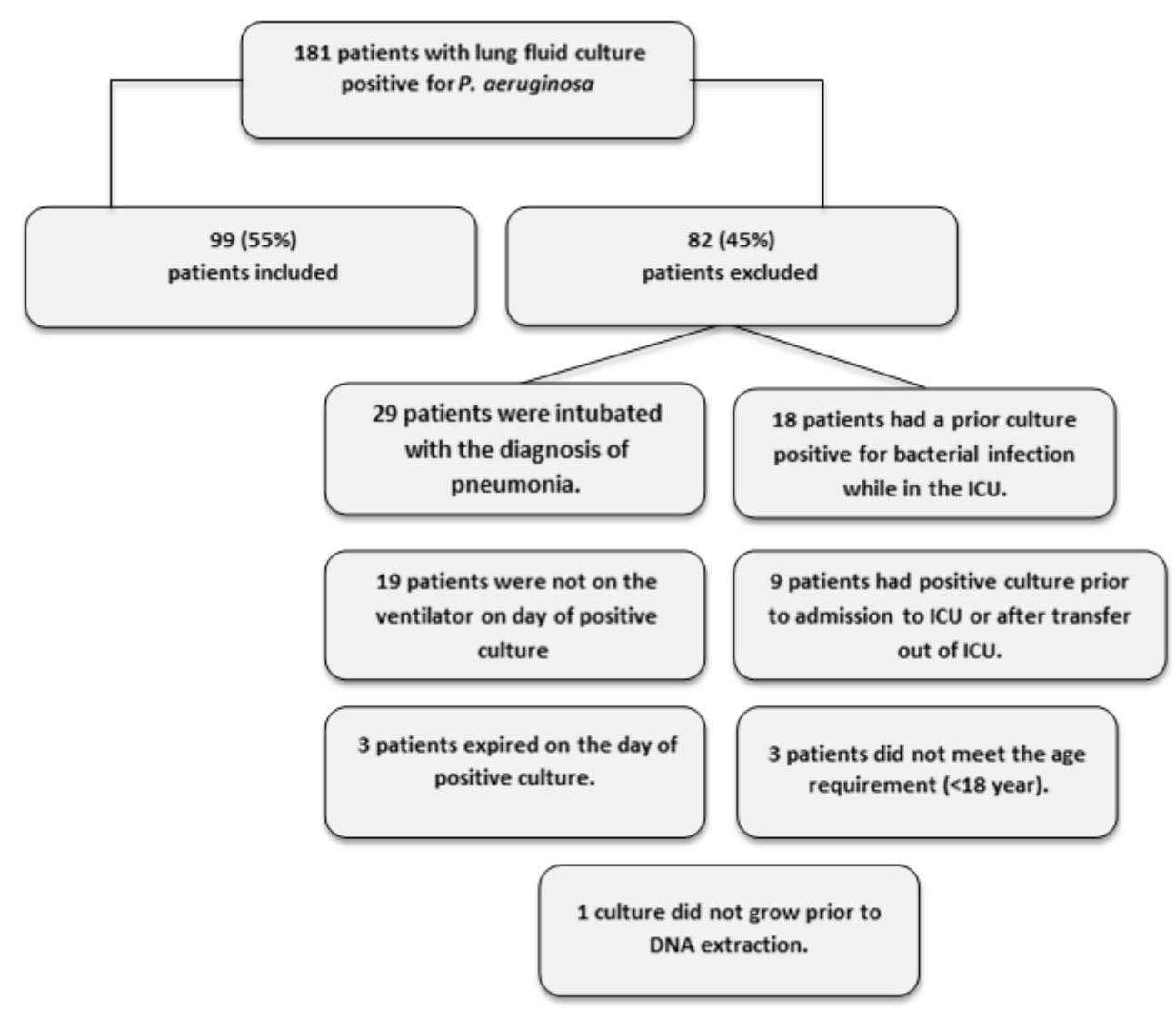

Figure 1. Flowchart of patient screening. Specific reasons for exclusion from the study can be found in the lower right hand side of the figure. 
Table 1. Patient demographics and characteristics. For each category, the number (n) and percentage $(\%)$ of patients (where applicable) in that category are provided.

\begin{tabular}{cc}
\hline Characteristics & n (\%) \\
\hline Total & 99 \\
Age in years & 57 \\
Gender (Male) & $68(68)$ \\
\hline Admission Type & \\
Trauma & $49(49)$ \\
Other & $50(50)$ \\
\hline Race & \\
White & $63(63)$ \\
Black & $30(30)$ \\
Hispanic & $6(6)$ \\
\hline Alcohol Consumer & \\
Current & $33(33)$ \\
Former & $3(3)$ \\
Unknown & $9(9)$ \\
\hline Smoking & \\
Current & $29(29)$ \\
Former & $22(22)$ \\
Unknown & $9(9)$ \\
\hline Illicit Drug Use & \\
Current & $11(11)$ \\
Former & $4(4)$ \\
Unknown & $12(12)$ \\
\hline
\end{tabular}

Table 2. Patient clinical characteristics. For injury scores or measures of end-organ dysfunction, both the median and the range are provided. Definitions of acronyms can be found in the Results Section. Of note, the 4 Point Lung Injury Score was not available for two patients, and the Trauma ISS was only available for 27 patients.

\begin{tabular}{cc}
\hline Injury Scores & Median (Range) \\
\hline APACHE II & $16(3-37)$ \\
SOFA & $7(0-16)$ \\
SAPS II & $35(13-67)$ \\
ODIN & $1(0-6)$ \\
4 Point Lung Injury & $1.67(0.33-3.33)$ \\
Trauma ISS & $18(4-45)$ \\
\hline Characteristics & Median (Range) \\
\hline Duration of Mechanical Ventilation, median & $21(3-118)$ \\
Length of ICU Stay & $24(3-118)$ \\
Time from Ventilation until Onset of pneumonia, median & $8(0-52)$ \\
\hline
\end{tabular}

\subsection{ExoY is More Prevalent than ExoU in Patients with P. aeruginosa Pneumonia}

To determine the prevalence of T3SS exoenzymes in our clinical P. aeruginosa isolates, a genotype analysis was performed (Table 3). There were 20/99 patients whose P. aeruginosa isolates have the ExoU. The prevalence of ExoY was high, present in 92/99 patients. Interestingly, the prevalence of ExoY without ExoU $\left(\right.$ ExoY $^{+} /$ExoU $\left.^{-}\right)$, hereafter referred to as 'ExoY only', was also high in ICU patients compared to ExoU without ExoY $\left(\right.$ ExoY $^{-} /$ExoU $\left.^{+}\right)$, hereafter referred to as 'ExoU only'; 74 versus two patients, respectively. Five patients had neither ExoU nor ExoY (ExoY $\left.{ }^{-} / \mathrm{ExoU}^{-}\right)$. 
Table 3. Relationship between presence of exoenzymes, mortality and drug resistance. For these data, isolates were grouped into the presence and/or absence of ExoY and ExoU. Both number (n) and percentage (\%) of isolate groups that are drug resistant, died or survived the length of the study have been provided.

\begin{tabular}{lccccc}
\hline & Isolates (n) & $\begin{array}{c}\text { Single-Drug } \\
\text { Resistant (n, \%) }\end{array}$ & $\begin{array}{c}\text { Multi-Drug } \\
\text { Resistant (n, \%) }\end{array}$ & $\begin{array}{c}\text { Died } \\
(\mathbf{n}, \mathbf{\%})\end{array}$ & Survived (n, \%) \\
\hline ExoY $^{+} / \mathrm{ExoU}^{-}$ & 74 & $6(8.1)$ & $13(17.6)$ & $17(23)$ & $57(77)$ \\
ExoY $^{-} / \mathrm{ExoU}^{+}$ & 2 & $0(0)$ & $0(0)$ & $0(0)$ & $2(100)$ \\
ExoY $^{+} / \mathrm{ExoU}^{+}$ & 18 & $0(0)$ & $3(16.7)$ & $3(16.7)$ & $15(83.3)$ \\
ExoY $^{-} / \mathrm{ExoU}^{-}$ & 5 & $2(40)$ & $1(20)$ & $2(40)$ & $3(60)$ \\
All Isolates & 99 & $8(8.1)$ & $17(17)$ & $22(22)$ & $77(78)$ \\
\hline
\end{tabular}

\subsection{Clinical Isolates Exhibit ExoU PLA 2 Enzyme Activity In Vitro and During the Infection of Cultured PMVECs}

To determine whether clinical isolates from BAL fluid utilize ExoU and/or ExoY as part of their virulence arsenal, we performed functional studies assessing enzyme activity (Figure 2). Using an in vitro $\mathrm{PLA}_{2}$ activity assay, we showed significant enzymatic activity in all clinical strains which possess ExoU; however, variable activity among these strains was noted. PA103 is a reference lab strain known to encode a functional ExoU. This strain was initially isolated from a patient $[26,27]$ and has been utilized extensively over the years as an ExoU ${ }^{+}$strain. In Figure 2A, we have delineated which samples are ExoU-positive or -negative by listing the exoenzymes which are present. All strains that begin with "JA" are clinical isolates from our study, and all other strains are lab strains. In subsequent figures, we refer to these isolates as $\mathrm{ExoU}^{+}$or ExoU ${ }^{-}$for clarity. When compared to PA103, JA810 (one of the ExoU ${ }^{+}$clinical strains isolated during our study) displayed higher PLA ${ }_{2}$ activity, whereas other clinical isolates had, to varying degrees, lower ExoU activity (Figure 2A). In contrast, none of the ExoU ${ }^{-}$clinical isolates displayed phospholipase activity. As a negative control, we included a PA103 mutant devoid of ExoU ( $\triangle$ UT mutant). Thus, these data confirm our PCR genotyping assay and demonstrate that ExoU ${ }^{+}$strains obtained from our patient cohort have $\mathrm{PLA}_{2}$ activity in vitro, whereas ExoU${ }^{-}$strains from these patients do not.

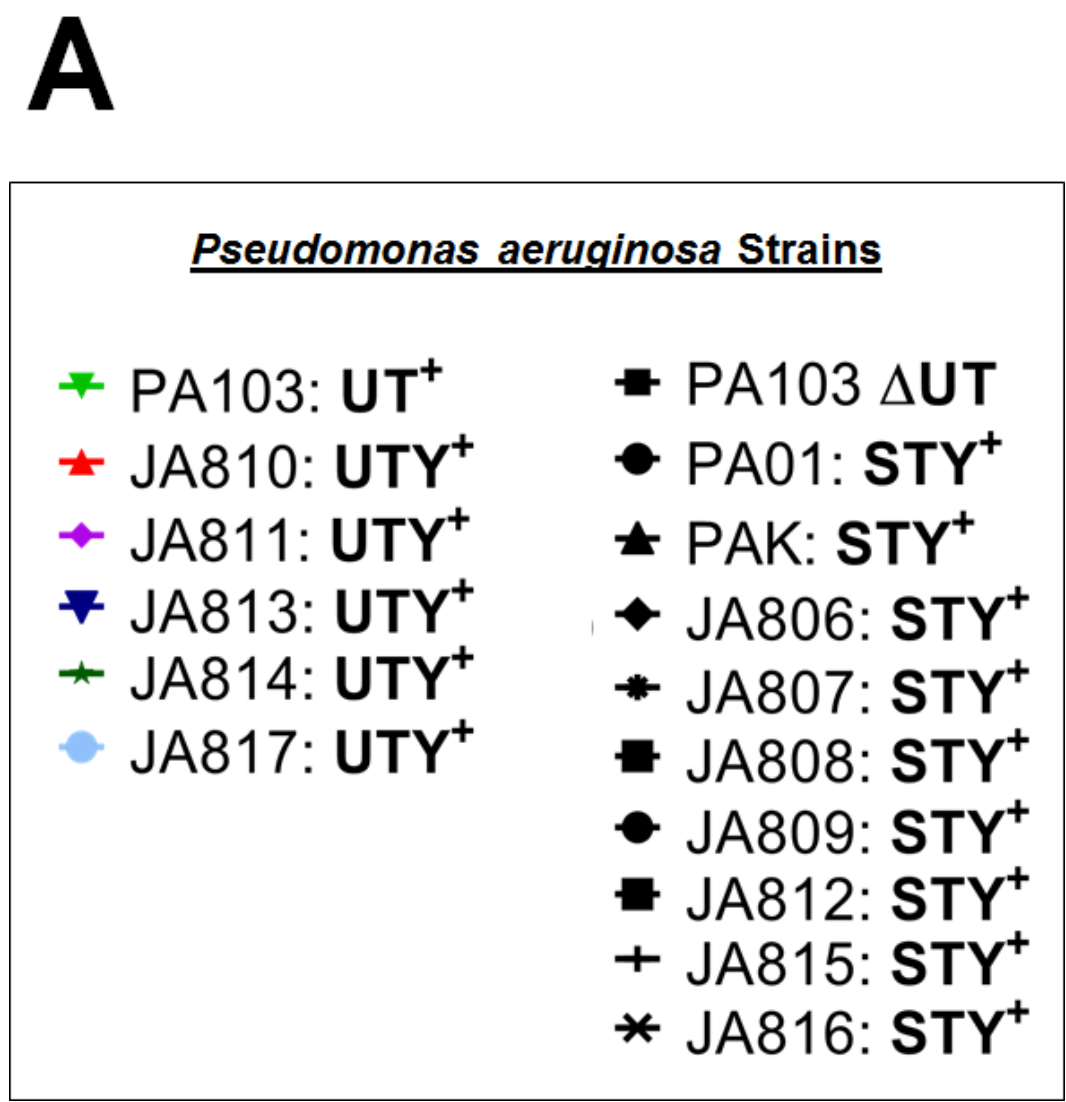

Figure 2. Cont. 

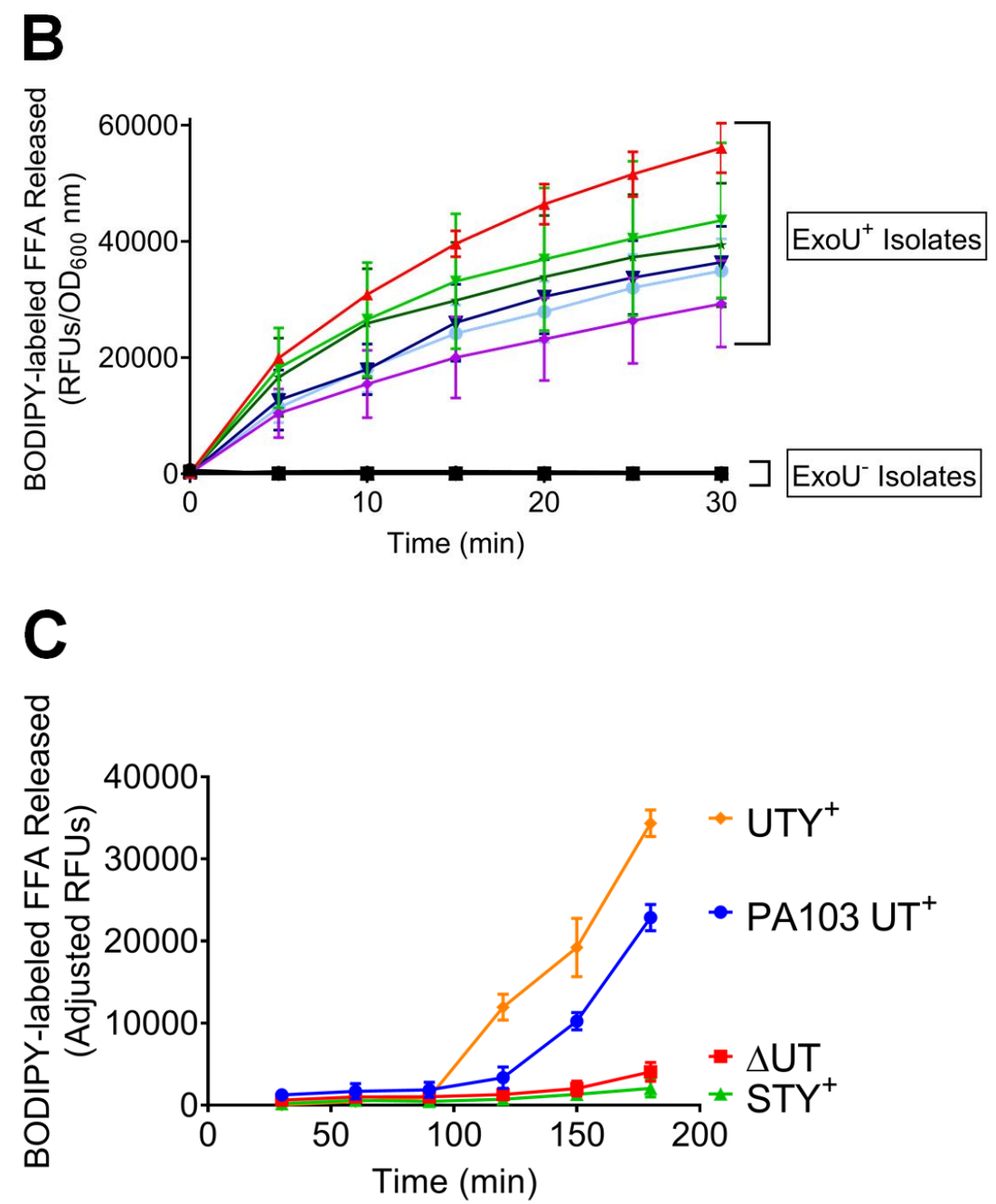

Figure 2. ExoU expression in bacterial isolates. (A) Bacterial cultures were selected randomly from patient isolates or lab strains known to possess ExoU per genotyping and grouped into ExoU ${ }^{+}$or ExoU ${ }^{-}$isolates for clarity. (B) Bacterial cultures were grown overnight. Single colonies were selected for inoculation into media with or without nitrilotriacetic acid (NTA) to induce the T3SS and secretion of exoenzymes to the culture medium. After overnight incubation, clarified supernatants were incubated with the fluorogenic PLA 2 substrate PED6, and enzyme activity was measured with a spectrofluorometer. Data in the assay are expressed as relative fluorescent units (RFU) normalized to the OD600 of the overnight culture. Data at each time point are expressed as mean +/- SEM, run in triplicate, $p<0.05$ comparing ExoU-positive isolates at $30 \mathrm{~min}$ to ExoU-negative isolates. (C) Confluent PMVECs were labeled with the PED6 substrate and subsequently exposed to bacteria with or without ExoU (at a MOI of 40:1). Supernatants were sampled over time to determine the presence of FFA using a spectrofluorometer. Data in the assay are expressed as relative fluorescent units (RFU), normalized to the OD600 of the overnight culture. Data at each time point are expressed as mean +/- SEM, run in triplicate, $p<0.05$ comparing ExoU-positive isolates at $180 \mathrm{~min}$ to ExoU-negative isolates.

We next sought to confirm these in vitro phospholipase $\mathrm{A}_{2}\left(\mathrm{PLA}_{2}\right)$ assay results in an infection assay using cultured pulmonary microvascular endothelial cells (PMVEC) as a target host cell. We used PMVECs because pneumonia leads to increased alveolar-capillary permeability with exudative edema, commonly involving hemorrhage. In response to the complex inflammatory milieu generated during infection, capillary endothelial cells lose cell-matrix and cell-cell attachments, leading to a breach in barrier integrity. In some cases, cell retraction progresses to apoptosis and/or necrosis, which impair vascular recovery following infection. Bacteria virulence factors, including T3SS effectors, 
act on capillary endothelium and promote barrier disruption. Both PA103 and an ExoU ${ }^{+}$clinical isolates generated the time-dependent accumulation of free fatty acid (FFA), indicating that ExoU was introduced from the bacteria into the host cell, where it activated phospholipase activity (Figure 2B). In contrast to ExoU-competent strains, the $\Delta \mathrm{UT}$ mutant, in which exoenzymes $\mathrm{T}$ and $\mathrm{U}$ have been deleted from PA103, was without activity, and an ExoU ${ }^{-}$clinical isolate displayed no activity. Overall, these findings support the assertion that clinical isolates possessing the ExoU gene have phospholipase $\mathrm{A}_{2}$ activity in an infection model. Similarly, ExoU deficient strains possess no phospholipase $\mathrm{A}_{2}$ activity.

\subsection{Clinical Isolates Have ExoY Activity in PMVECs}

We next examined ExoY activity in 'ExoY only' and ExoY-negative clinical isolates. ExoY is a promiscuous cyclase which generates both canonical (cAMP and cGMP) and non-canonical (cCMP and cUMP) cyclic nucleotides. Here, we measured cellular cGMP levels at $6 \mathrm{~h}$ post-infection, as cGMP was produced first and to the largest extent, after the infection with PMVECs infected with a lab strain expressing exogenous ExoY, as reported previously [14]. Our data indicate that 'ExoY only' clinical isolates can induce cGMP production in PMVECs (Figure 3). Compared to uninfected PMVECs or PMVECs infected with ExoY-negative clinical strains, they do so with highly variable activity, ranging from 200 to 2000 pmol cGMP per mg protein in the six strains tested. These data illustrate that the enzyme entered the endothelium, acquiring its functional tertiary structure and interacting with its necessary co-factor, which is thought to be actin [17]. Thus, ExoY competent clinical strains utilize ExoY as a part of their virulence arsenal. They are able to introduce an enzyme that acquires activity in host cells.

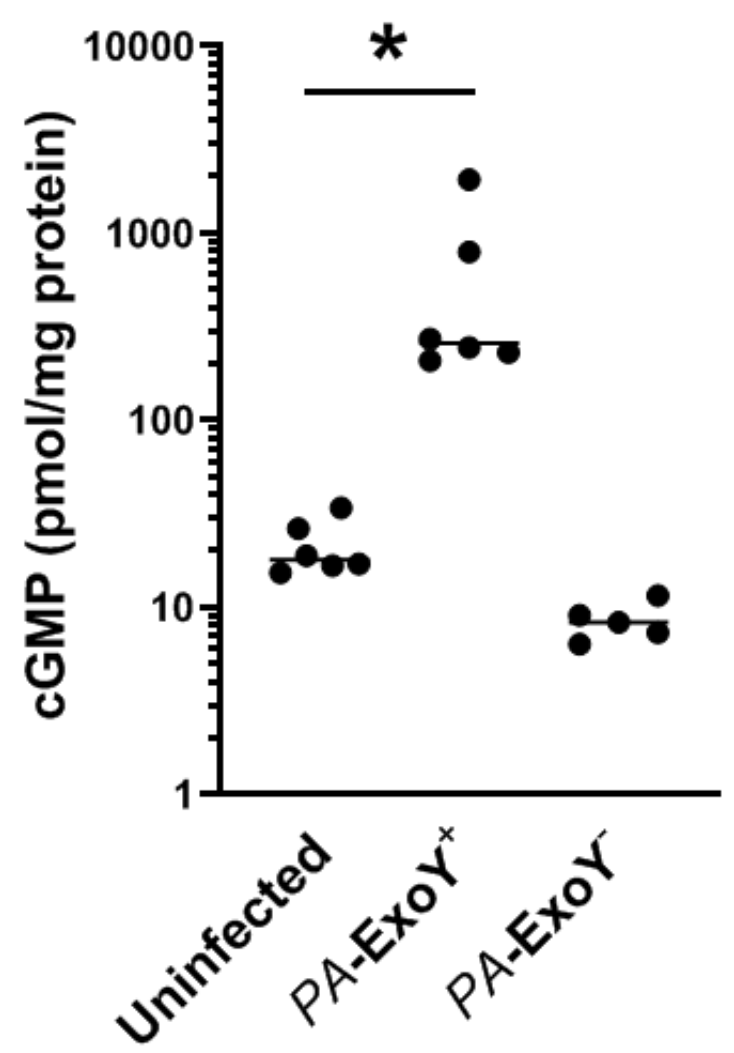

Figure 3. ExoY expression in bacterial isolates. Confluent layers of PMVECs were exposed for $6 \mathrm{~h}$ to distinct ExoY-positive $\left(\right.$ ExoY $^{+} /$ExoU $\left.^{-}\right)$or ExoY-negative $\left(\right.$ExoY $\left.^{-}\right)$strains at an $\mathrm{MOI}$ of 40:1, after which cells were harvested and intracellular cGMP levels were measured by enzyme immuno assay. Data are expressed as pmol cGMP per mg cell protein and are compared to uninfected PMVECs. ${ }^{*} p<0.05$ when comparing $P A$-infected cells at $6 \mathrm{~h}$ to uninfected PMVEC. 


\subsection{End-Organ Injury Attributable to ExoY and ExoU}

To better understand how ExoY may affect patient morbidity, we evaluated whether the presence of ExoY was associated with the development of acute kidney injury (AKI), cardiovascular dysfunction (CV), or coagulopathy (Table 4). Definitions of these measures of end-organ dysfunction can be found in the Methods section. We evaluated all patients in the study and then separately analyzed patients with isolates containing ExoY without ExoU $\left(\mathrm{ExoY}^{+} / \mathrm{ExoU}^{-}\right)$and patients with isolates positive for ExoU (with and without ExoY) (Table 4). Of note, data were not available for all patients or could not be measured for reasons listed in the Methods section. For AKI as a measure of end-organ dysfunction, 42/94 patients in the study were diagnosed with AKI in the week after a diagnosis of pneumonia. Of patients harboring ExoY only isolates, 35/71 had AKI and 6/18 patients harboring ExoU isolates had AKI. Regarding cardiovascular dysfunction, $42 / 99$ patients in the study required vasopressors to support their blood pressure and maintain perfusion to critical end organs in the week after a diagnosis of pneumonia. For patients who were infected with ExoY only isolates, 28/74 required vasopressors, compared to 6/20 patients infected with ExoU isolates. Finally, 72/92 patients in the study had coagulopathy after their diagnosis of pneumonia. For patients with ExoY only isolates, $54 / 70$ had coagulopathy compared to 14/17 patients with ExoU isolates. There is a clinical trend in the AKI group (but no statistical significance, $p=0.225$ ) when comparing ExoY alone to ExoU towards more AKI when patients have isolates with ExoY only. Patients with ExoY only isolates had slightly more $\mathrm{CV}$ dysfunction compared to patients with clinical strains positive for ExoU, and patients with ExoY only isolates had slightly less coagulopathy compared to patients with isolates positive for ExoU. Importantly, the presence of ExoY alone is associated with end-organ dysfunction.

Table 4. Relationship between end-organ dysfunction in all patients, patient with ExoY ${ }^{+} / \mathrm{ExoU}^{-}$and patients with ExoU only. For AKI, five patients (three in the ExoY ${ }^{+} /$ExoU $^{-}$group) were excluded as they had a diagnosis of end-stage renal disease. For coagulopathy, seven patients (four in the ExoY $\mathrm{Y}^{+} \mathrm{ExoU}^{-}$ group) did not have any data available during the timeframe examined.

\begin{tabular}{cccc}
\hline & All Patients & $\begin{array}{c}\text { Exơ }{ }^{+} / \text {ExoU }^{-} \\
\text {Patients }\end{array}$ & All ExoU $^{+}$Patients \\
\hline Acute Kidney Injury & $42 / 94$ & $35 / 71$ & $6 / 18$ \\
CV dysfunction & $42 / 99$ & $28 / 74$ & $6 / 20$ \\
Coagulopathy & $72 / 92$ & $54 / 70$ & $14 / 17$ \\
\hline
\end{tabular}

\subsection{Mortality and Drug Resistance in ExoY $Y^{+}$Clinical Isolates}

Pneumonia caused by ExoU expressing P. aeruginosa is known to be associated with illness severity, high mortality and antibiotic drug resistance [25]. However, the importance of the presence of ExoY by $P$. aeruginosa for illness severity and mortality remains unknown. Because only 2 patients in our cohort had pneumonia with ExoY ${ }^{-} / \mathrm{ExoU}^{+}$P. aeruginosa, we could not statistically compare the mortality associated with the presence of ExoY without ExoU in our study with the mortality associated to ExoU. However, 17/74 patients with ExoY ${ }^{+} / \mathrm{ExoU}^{-}$clinical strains died and 20/92 patients with any ExoY (ExoU-positive and -negative) died, with rates of $23 \%$ and $21.7 \%$, respectively (Table 3). Furthermore, we identified clinical isolates with either single- or multi-drug resistance. In this study, 6/74 (8.1\%) of patients with ExoY ${ }^{+} /$ExoU $^{-}$clinical strains and 6/92 (6.5\%) of patients with any ExoY had single-drug resistance. Interestingly, multi-drug resistance increased for patients compared to single-drug resistance; $13 / 74(17.6 \%)$ and 16/92 (17.4\%) had multi-drug resistance in ExoY ${ }^{+} /$ExoU $^{-}$ clinical strains and patients with any ExoY, respectively.

\section{Discussion}

P. aeruginosa is one of the major bacterial pathogens that causes acute pulmonary infections and is associated with significant morbidity and mortality in critically ill and immunocompromised 
patients [9]. Among the toxins produced by P. aeruginosa, preclinical studies have shown that T3SS exoenzymes play a major role in the virulence of that bacterium [6-10]. There is thus a growing interest in determining whether there is an association between the genetic presence of these T3SS exoenzymes and the outcome of critically ill patients who develop infection with P. aeruginosa [28-30]. Of the four exoenzymes, the presence of ExoU has previously been shown to be associated with drug resistance and increased mortality in patients with P. aeruginosa pneumonia [6,31].

ExoY is a promiscuous cyclic nucleotidyl cyclase capable of generating canonical (cAMP and cGMP) and non-canonical (cUMP and cCMP) cNMPs. The ability to generate each of these cNMPs differs among cell types, although at this point, the mechanisms responsible for discriminating which cNMPs are produced remain unknown [14]. Nonetheless, these cNMPs activate protein kinase A, resulting in phosphorylation of multiple (incompletely described) effectors, including tau. Tau hyperphosphorylation results in its dissociation from microtubules leading to tubule collapse, which in turn elicits cell rounding [32]. These cellular events strictly require ExoY enzymatic activity, i.e., the ability to produce cNMPs.

It is, however, unknown whether ExoY may also cause morbidity and mortality in critically ill patients who develop P. aeruginosa pneumonia [12]. To determine whether ExoY was associated with patient morbidity and mortality, we recruited ICU patients with primary P. aeruginosa pneumonia to determine the prevalence of ExoY and correlate the presence of ExoY with mortality, drug resistance and the development of end-organ dysfunction in these patients. The presence of ExoY without the presence of ExoU was detected in the vast majority of isolates. Overall, 75\% of the isolates were $\mathrm{ExoY}^{+} / \mathrm{ExoU}^{-}$and only $2 \%$ were $\mathrm{ExoY}^{-} / \mathrm{ExoU}^{+}$. The results of the present study demonstrate that patients with ExoY $^{+} /$ExoU $^{-}$isolates have significant mortality (23\%) and single- or multi-drug resistance $(8.1 \%$ and $17.6 \%$, respectively). There is no way to statistically compare these data to patients harboring ExoU ${ }^{+}$clinical isolates because the numbers are too few in this study. Additionally, prior studies examining ExoU and mortality did not examine ExoY at the time of the study; therefore, reasonable statistical comparisons are not available. However, this is the first study to report mortality and drug resistance for patients with ExoY-containing isolates. Our study suggests that ExoY is likely a contributor to patient morbidity and mortality based on the percentage of patients that died due to infection with ExoY-positive isolates.

In the last few decades, survival from critical illness (i.e., sepsis, septic shock, acute respiratory distress syndrome, etc.) has improved; however, in more recent years, the survival numbers have stabilized and no pharmacotherapy has been found to further improve survival [33-37]. Interestingly, while 28-day survival has improved for many patients, mortality at 1-2 years for these survivors is staggering [38-40]. Additionally, acute critical illness is now becoming chronic and patients are surviving with increased morbidity. Therefore, while measurement of mortality remains paramount, measurement of end-organ dysfunction is moving to the forefront as a mechanism of long-term morbidity. In our study, patients with ExoY-positive clinical isolates had coagulopathy and cardiovascular dysfunction, although it was not significantly different than ExoU ${ }^{+}$isolates. There was a trend, though statistically insignificant, towards AKI. This is likely a Type II error, as more patients recruited to the study would have expanded this difference to statistical significance. How ExoY and/or ExoU contribute to these outcomes in end-organ dysfunction is currently unclear, but is the source of intense investigation.

Although mechanism(s) of end-organ dysfunction in survivors of critical illness remain unclear, recent preclinical studies demonstrate that pulmonary endothelial cell infection with P. aeruginosa causes extracellular release of oligomerized tau and amyloids in an ExoY- and ExoU-dependent manner [23,41]. First, oligomerized tau was released to the extracellular space after phosphorylation and degradation from microtubules. Furthermore, this tau was insoluble, could not be degraded with most techniques (resistance to enrichment with 50\% ammonium sulfate precipitation and insensitivity to boiling, detergents, proteases, RNases and DNases) and was transmissible and propagated in naïve endothelial cells. Interestingly, tau was detected in the bronchoalveolar lavage fluid from patients with P. aeruginosa 
pneumonia and, when added to pulmonary endothelial cell monolayers, caused increased permeability in a dose-dependent fashion [41]. Additionally, amyloids were released after pulmonary endothelial cell infection with $P$. aeruginosa. Like tau, these amyloids demonstrated the ability to propagate in naïve endothelial cells and also displayed some insensitivity to degradation, indicating the behavior of a prion-like entity. In addition to being detected in the bronchoalveolar lavage fluid of patients with P. aeruginosa, they also disrupt long-term potentiation in the rat hippocampus [22,23]. Thus, generation of transmissible and cytotoxic amyloids within the lung may contribute to end-organ dysfunction that is due to ExoY and ExoU. Here, we report that patients infected with bacteria possessing ExoY were associated with significant cardiovascular events and coagulopathy; furthermore, we demonstrate a clinical trend towards more AKI and patients with ExoY clinical isolates. Future studies will address whether distinct amyloid species generated by ExoY and ExoU, respectively, account for this difference in end organ dysfunction.

This is the first study to report mortality and drug resistance for patients with ExoY-containing isolates, and the percentage of patients that died in this study with ExoY-positive isolates and pre-clinical studies indicate that ExoY is likely a contributor to patient morbidity and mortality. Limitations to our study include an inability to detect a difference in mortality between ExoY ${ }^{+} / \mathrm{ExoU}^{-}$and ExoY $\mathrm{Y}^{-} / \mathrm{ExoU}^{+}$ isolates, given the discrepancy in the frequency of each within the study population. Furthermore, we have not screened ExoU activity or ExoY-dependent cyclic nucleotide levels in all 99 patient clinical isolates included in this study and hence cannot correlate the amount of exoenzymes produced in the patients with their health outcomes. However, detailed biochemical analysis of the strain-by-strain ExoU and ExoY activity is beyond the scope of the current manuscript.

In conclusion, ExoY is associated with patient mortality and end-organ dysfunction. In addition to ExoU, ExoY appears to be another virulence factor of $P$. aeruginosa pneumonia in critically ill patients. Future studies should further investigate the relative importance of ExoY protein expression as an important $P$. aeruginosa virulence factor and consider new treatments for inhibiting injection of T3SS toxins into parenchymal lung cells to prevent end-organ damage after P. aeruginosa bacteria are cleared from the lung by antibiotic therapy.

\section{Conclusions}

ExoY contributes to patient end-organ dysfunction. In addition to ExoU, ExoY appears to be another virulence factor of $P$. aeruginosa pneumonia in critically ill patients. Future studies should further investigate the relative importance of ExoY protein expression as an important $P$. aeruginosa virulence factor and consider new treatments for preventing the injection of T3SS toxins into parenchymal lung cells which continue to cause end-organ damage after $P$. aeruginosa bacteria are cleared from the lung by antibiotic therapy.

\section{Materials and Methods}

\subsection{Study Design}

Our study was a single-center, prospective observational study conducted at the University of Alabama at Birmingham (UAB) Hospital between November 2012 and January 2015. Patients were recruited from any of four intensive care units (Trauma-Burn Intensive Care Unit (ICU), Neuro ICU, Surgical ICU, or Medical ICU) at UAB hospital. All mechanically ventilated patients with a bronchoalveolar lavage (BAL) fluid culture positive for P. aeruginosa were considered if the infection was the patient's first infection. Exclusion criteria included an age of less than 18 years, any prior positive culture from any source, pre-existing pneumonia on admission and/or prior to intubation, prisoners directly admitted from a correctional facility, and patients enrolled in an ongoing, interventional and randomized clinical trial. Patients were followed from ICU admission to ICU discharge and clinical data were prospectively collected on all patients enrolled in the study. For this study, we obtained an Exclusion of Informed Consent, as the bacterial isolates collected were considered 'remnant tissue' 
and no protected health information was obtained from the enrollees. This study was conducted in accordance with the Declaration of Helsinki and the protocol was approved by The University of Alabama at Birmingham Institutional Review Board (Protocol Number-X120513006, original approval date was July 19, 2012. The study remains under current approval).

\subsection{Isolation and Identification of Clinical P. aeruginosa}

BAL samples, submitted to the microbiology laboratory for bacterial culture, were vortexed for 30-60 s, followed by the inoculation of Trypticase Soy Agar II with 5\% Sheep Blood, Chocolate Agar II and MacConkey Agar plates (Becton Dickinson, Sparks, MD, USA). Plates were then placed in an incubator containing approximately $5 \% \mathrm{CO}_{2}$ at $35{ }^{\circ} \mathrm{C}$ for $24-48 \mathrm{~h}$. Colonies from the quantitative primary media were counted, where each colony $=1000$ colony-forming units (CFU) per milliliter [42]. Isolated colonies were identified by matrix assisted laser desorption ionization time of flight mass spectroscopy (MALDI-TOF, Vitek ${ }^{\circledR}$ MS, Biomerieux). Multiple stocks for each bacterial isolate were created by storing bacteria in $30 \%$ glycerol with media and keeping the stocks at $-80{ }^{\circ} \mathrm{C}$. These stocks were continuously used for this work; therefore, genetic drift due to continual bacterial growth is minimized.

\subsection{PCR Method and Gel Electrophoresis}

Clinical isolates of P. aeruginosa were genotyped by PCR using GoTaq Green 2× PCR master mix (Promega, Madison, WI, USA). Each $25 \mu \mathrm{L}$ reaction contained 50-100 ng of P. aeruginosa genomic DNA and $0.5 \mu \mathrm{M}$ concentration of each PCR primer. PCR primers were mixed into two groups, and forward and reverse sequences are listed in Table $5(\mathrm{Mix} 1=\operatorname{exoU}+\operatorname{exoT}+\operatorname{pol} A, \operatorname{Mix} 2=\operatorname{exoS}+\operatorname{exo} Y+\operatorname{pol} A)$. The polA gene was used as the internal control in all reactions. Initial denaturation was performed at $94{ }^{\circ} \mathrm{C}$ for $5 \mathrm{~min}$, followed by 35 cycles of denaturation at $94{ }^{\circ} \mathrm{C}$ for $30 \mathrm{~s}$, annealing at $55^{\circ} \mathrm{C}$ for $20 \mathrm{~s}$ and extension at $72{ }^{\circ} \mathrm{C}$ for $50 \mathrm{~s}$, followed by final extension at $72{ }^{\circ} \mathrm{C}$ for $3 \mathrm{~min}$ and holding at $4{ }^{\circ} \mathrm{C}$. PCR products were run on $1 \%$ agarose gel, stained with ethidium bromide and imaged using a BioDoc-it system. The four exoenzymes genotyped were ExoS, ExoT, ExoU, and ExoY.

Table 5. PCR sequences for exoenzyme genotyping. For each gene, the $5^{\prime}$ to $3^{\prime}$ sequence of the forward and reverse primers are listed. Expected band size for each PCR fragment generated is listed.

\begin{tabular}{ccc}
\hline Primer Name & Primer Sequence & PCR Fragment Size \\
\hline exoY forward & TGAGCGAGGACGGATTCTA & $309 \mathrm{bp}$ \\
\hline exoY reverse & GATAGCCGTTGCCCTTGAT & \\
\hline exoU forward & CTCAATGTACTCCCACGCATAG & $406 \mathrm{bp}$ \\
\hline exoU reverse & CATCCTGGAATTCTGTCCACTC & \\
\hline exoT forward & GCCGAGATCAAGCAGATGAT & $405 \mathrm{bp}$ \\
\hline exoT reverse & GACAGGCTCGCCCTTTAC & \\
\hline exoS forward & CATCAGGTAATGAGCGAGGTC & \\
\hline exoS reverse & TTCAGGGAGGTGGAGAGATAG & $707 \mathrm{bp}$ \\
\hline PolA forward & TTTCCTGCAGCCAGTTATCC & \\
\hline PolA reverse & CAAGCTCAAGAGCACCTACA & \\
\hline
\end{tabular}

\subsection{Measurement of Secreted ExoU Activity In Vitro}

Multiple patient isolates that were ExoU ${ }^{+}$or ExoU ${ }^{-}$were randomly selected and screened for the presence of ExoU PLA 2 enzymatic activity in order to validate the PCR results. We used a previously established assay by which bacteria grown in liquid culture can be triggered to produce and secrete T3SS effectors into the culture medium via the chelation of divalent cations [43]. To do this, bacterial 
cultures were grown from frozen stocks onto LB agar medium at $37^{\circ} \mathrm{C}$ overnight. Single colonies were inoculated into Trypticase Soy Broth (TSB) liquid cultures supplemented with monosodium glutamate $(0.1 \mathrm{M})$, glycerol $(1 \%)$ and nitrilotriacetic acid (NTA, $0.01 \mathrm{M})$ (T3SS activation) or without NTA (no T3SS activation) and grown overnight with aeration at $32{ }^{\circ} \mathrm{C}$. Under the + NTA conditions, exoenzymes are secreted directly into the culture medium. The optical density at $600 \mathrm{~nm}$ (OD600) was measured in the overnight liquid cultures for $\mathrm{PLA}_{2}$ assay normalization. Bacteria were removed from liquid overnight cultures by centrifugation and clarified supernatants (i.e., bacteria free) were used to measure ExoU $\mathrm{PLA}_{2}$ activity. PLA 2 activity assays contained $14.5 \mu \mathrm{L}$ of supernatant in a buffer solution containing $50 \mathrm{mM}$ MOPS (pH 7.4) and $50 \mathrm{mM} \mathrm{NaCl}$. To each reaction, we then added a fluorogenic PLA 2 substrate, PED6 (N-((6-(2,4-Dinitrophenyl)amino)hexanoyl)-2-(4,4-Difluoro-5,7-Dimethyl-4-Bora-3a,4a-Diaza-sIndacene-3-Pentanoyl)-1-Hexadecanoyl-sn-Glycero-3-Phosphoethanolamine, Triethylammonium Salt, $30 \mu \mathrm{M}$ ) followed by incubation at room temperature for $30 \mathrm{~min}$. Subsequently, poly-Ubiquitin $(0.1 \mathrm{mg} / \mathrm{mL})$ was added to activate ExoU PLA 2 activity [44] and samples were removed every $5 \mathrm{~min}$. Negative control reactions did not have poly-Ubiquitin added. ExoU PLA $\mathrm{P}_{2}$ activity results were generated by quantifying the release of BODIPY-labeled free fatty acid (FFA) from PED6 hydrolysis $[45,46]$ using a nano-spectrofluorometer. Data are expressed as relative fluorescent units (RFU) normalized to the OD600 of the overnight culture.

\subsection{Rat Pulmonary Microvascular Endothelial Cell Isolation and Culture}

Rat pulmonary microvascular endothelial cells (PMVEC) were isolated as described [47]. Harvested cells were transferred to a growth medium and primary cultures were characterized by cobblestone morphology and endothelial cell markers. PMVECs were cultured in DMEM 10\% FBS and $1 \%$ penicillin/streptomycin at $37^{\circ} \mathrm{C}$ and $5 \% \mathrm{CO}_{2}$.

\subsection{Measurement of ExoU Activity in PMVECs}

Bacterial cultures were again grown from frozen stocks onto minimal $\mathrm{E}$ salts agar medium at $37^{\circ} \mathrm{C}$ overnight. They were scraped into $10 \mathrm{~mL}$ sterile normal saline, collected by centrifugation, resuspended into $1 \mathrm{~mL}$ of saline and the OD600 was then measured. Confluent PMVEC monolayers were seeded at confluence prior to bacterial inoculation as previously described [48]. PMVECs were washed with serum- and phenol red-free DMEM and incubated in DMEM containing PED6 $(30 \mu \mathrm{M})$ fluorogenic substrate for $1 \mathrm{~h}$ at $37^{\circ} \mathrm{C}, 5 \% \mathrm{CO}_{2}$. After $1 \mathrm{~h}$, media were removed from the cells and replaced with fresh DMEM-containing bacteria at a multiplicity of infection (MOI) of 40:1. Medium samples were removed from the culture at the times indicated and immediately read on the nano-spectrofluorometer to determine the amount of BODIPY-FFA released during infection. A control well of cells labeled with PED6, but without infection, was sampled over the same time course, and these values for each time point were subtracted from the corresponding infection (experimental) samples to normalize the data for the endogenous background release of BODIPY-FFA from the host cells. Data are expressed as normalized RFUs.

\subsection{Measurement of ExoY Activity in PMVECs}

Multiple patient isolates that were $\mathrm{ExoY}^{+} / \mathrm{ExoU}^{-}$and $\mathrm{ExoY}^{-}$were randomly selected for the measurement of their ExoY activity. PMVECs were seeded and grown to confluence in 6-well plates. Bacterial strains were prepared for infection, as previously described [32], and bacteria were incubated on PMVECs at a MOI of 40:1. After 6 h, cells were washed, collected and cGMP was measured as previously described [14].

\subsection{Determination of End-Organ Dysfunction}

All patients were examined for acute kidney injury (AKI), cardiovascular dysfunction and coagulopathy after their diagnosis of P. aeruginosa pneumonia. For AKI, we used the Kidney Disease Improving Global Outcomes (KDIGO) definition for creatinine only [49]. After a diagnosis of primary 
pneumonia, if the patient's serum creatinine increased by $0.3 \mathrm{mg} / \mathrm{dL}$ over $48 \mathrm{~h}$ this was considered a positive diagnosis of AKI. Patients were evaluated in 48-h periods from the diagnosis of pneumonia for a total of 7 days. Patients were given a positive diagnosis of AKI if continuous renal replacement therapy was present in the week after the diagnosis of pneumonia. If a patient had a diagnosis of end-stage renal disease, they were excluded from this particular analysis. For CV dysfunction, we evaluated all patients for the presence of absence of a vasopressor in the week after their diagnosis of pneumonia. For coagulopathy, we evaluated patients' international normalized ratio (INR) for a week after a diagnosis of pneumonia. If, at any time, the INR was $\geq 1.2$, they were given a diagnosis of coagulopathy. Some patients did not have their INR analyzed during their ICU stay; these patients were excluded from this particular analysis.

\subsection{Statistical Analysis}

All demographic and clinical variables with continuous measures were expressed as means, and a $95 \%$ confidence interval and categorical variables were expressed as proportions. The Chi-square test was used to compare end-organ dysfunction with ExoY and ExoU in patients. For in vitro data, all normal data are mean \pm SEM. For normally distributed data, one-way ANOVA followed by Dunnett's test was used to compare three or more groups and Student's t-test to compare 2 groups. Bonferroni correction was used to adjust multiple comparisons. Statistical analyses were done with Prizm GraphPad. A $p$ value of $\leq 0.05$ was considered statistically significant.

Author Contributions: For this research manuscript, the following contributions were provided: conceptualization, B.M.W., T.S., and J.-F.P.; methodology, B.M.W., T.S. and J.-F.P.; validation, B.M.W., N.A., C.M. and J.-F.P.; formal analysis, B.M.W., N.A., J.P.A., W.R., C.M. and J.-F.P.; investigation, B.M.W., N.A., S.C.C., M.E.B., J.C.P, A.T.T., R.R.W., K.D.I., S.A.M., T.S., M.F.A., J.P.A., W.R., K.S.H., L.A.S., C.M.; resources, B.M.W., T.S., M.F.A., J.P.A., W.R., K.S., L.A.S. and J.-F.P.; data curation, B.M.W., N.A., and J.-F.P.; writing-original draft preparation, B.M.W. and J.-F.P.; writing—review and editing, B.M.W., N.A., S.C.C., M.E.B., J.C.P., A.T.T., R.R.W., K.D.I., S.A.M., T.S., M.F.A., J.P.A., W.R., K.S., L.A.S., C.M. and J.-F.P.; visualization, B.M.W., N.A. and J.-F.P.; supervision, B.M.W. and J.-F.P.; project administration, B.M.W. and J.-F.P.; funding acquisition, B.M.W., T.S., M.F.A., J.P.A., W.R., K.S., L.A.S. and J.-F.P. All authors have read and agreed to the published version of the manuscript.

Funding: This research was funded by NIH grants GM127584 (to B.M.W. and J.-F.P.), HL66299 and HL60024 (to T.S.), OD010944 (to M.A.), HL076125 (to L.A.S.), HL141473 (to W.R.). HL118334 (to J.P.A.), HL118334-S1 (to K.S.H. and J.P.A.) and DoD grant W81XWH-16-1-0096 (to M.A.).

Conflicts of Interest: The authors declare no conflict of interest. The funders had no role in the design of the study; in the collection, analyses, or interpretation of data; in the writing of the manuscript, or in the decision to publish the results.

\section{References}

1. Sawa, T.; Shimizu, M.; Moriyama, K.; Wiener-Kronish, J.P. Association between Pseudomonas aeruginosa type III secretion, antibiotic resistance, and clinical outcome: A review. Crit. Care 2014, 18, 668. [CrossRef]

2. Boucher, H.W.; Talbot, G.H.; Bradley, J.S.; Edwards, J.E.; Gilbert, D.; Rice, L.B.; Scheld, M.; Spellberg, B.; Bartlett, J. Bad bugs, no drugs: No ESKAPE! An update from the Infectious Diseases Society of America. Clin. Infect. Dis. 2009, 48, 1-12. [CrossRef]

3. Pendleton, J.N.; Gorman, S.P.; Gilmore, B.F. Clinical relevance of the ESKAPE pathogens. Expert Rev. Anti Infect. Ther. 2013, 11, 297-308. [CrossRef]

4. El Solh, A.A.; Akinnusi, M.E.; Wiener-Kronish, J.P.; Lynch, S.V.; Pineda, L.A.; Szarpa, K. Persistent infection with Pseudomonas aeruginosa in ventilator-associated pneumonia. Am. J. Respir. Crit. Care Med. 2008, 178, 513-519. [CrossRef] [PubMed]

5. Ertugrul, B.M.; Yildirim, A.; Ay, P.; Oncu, S.; Cagatay, A.; Cakar, N.; Ertekin, C.; Ozsut, H.; Eraksoy, H.; Calangu, S. Ventilator-associated pneumonia in surgical emergency intensive care unit. Saudi Med. J. 2006, 27, 52-57. [PubMed]

6. Pena, C.; Cabot, G.; Gomez-Zorrilla, S.; Zamorano, L.; Ocampo-Sosa, A.; Murillas, J.; Almirante, B.; Pomar, V.; Aguilar, M.; Granados, A.; et al. Influence of virulence genotype and resistance profile in the mortality of Pseudomonas aeruginosa bloodstream infections. Clin. Infect. Dis. 2015, 60, 539-548. [CrossRef] [PubMed] 
7. Roy-Burman, A.; Savel, R.H.; Racine, S.; Swanson, B.L.; Revadigar, N.S.; Fujimoto, J.; Sawa, T.; Frank, D.W.; Wiener-Kronish, J.P. Type III protein secretion is associated with death in lower respiratory and systemic Pseudomonas aeruginosa infections. J. Infect. Dis. 2001, 183, 1767-1774. [CrossRef]

8. Angus, A.A.; Evans, D.J.; Barbieri, J.T.; Fleiszig, S.M. The ADP-ribosylation domain of Pseudomonas aeruginosa ExoS is required for membrane bleb niche formation and bacterial survival within epithelial cells. Infect. Immun. 2010, 78, 4500-4510. [CrossRef] [PubMed]

9. Juan, C.; Pena, C.; Oliver, A. Host and Pathogen Biomarkers for Severe Pseudomonas aeruginosa Infections. J. Infect. Dis. 2017, 215, S44-S51. [CrossRef]

10. Wong-Beringer, A.; Wiener-Kronish, J.; Lynch, S.; Flanagan, J. Comparison of type III secretion system virulence among fluoroquinolone-susceptible and -resistant clinical isolates of Pseudomonas aeruginosa. Clin. Microbiol. Infect. 2008, 14, 330-336. [CrossRef]

11. Engel, J.; Balachandran, P. Role of Pseudomonas aeruginosa type III effectors in disease. Curr. Opin. Microbiol. 2009, 12, 61-66. [CrossRef] [PubMed]

12. Morrow, K.A.; Frank, D.W.; Balczon, R.; Stevens, T. The Pseudomonas aeruginosa Exoenzyme Y: A Promiscuous Nucleotidyl Cyclase Edema Factor and Virulence Determinant. Handb. Exp. Pharmacol. 2017, 238, 67-85. [CrossRef] [PubMed]

13. Kloth, C.; Schirmer, B.; Munder, A.; Stelzer, T.; Rothschuh, J.; Seifert, R. The Role of Pseudomonas aeruginosa ExoY in an Acute Mouse Lung Infection Model. Toxins 2018, 10, 185. [CrossRef] [PubMed]

14. Morrow, K.A.; Seifert, R.; Kaever, V.; Britain, A.L.; Sayner, S.L.; Ochoa, C.D.; Cioffi, E.A.; Frank, D.W.; Rich, T.C.; Stevens, T. Heterogeneity of pulmonary endothelial cyclic nucleotide response to Pseudomonas aeruginosa ExoY infection. Am. J. Physiol. Lung Cell. Mol. Physiol. 2015, 309, L1199-L1207. [CrossRef]

15. Munder, A.; Rothschuh, J.; Schirmer, B.; Klockgether, J.; Kaever, V.; Tummler, B.; Seifert, R.; Kloth, C. The Pseudomonas aeruginosa ExoY phenotype of high-copy-number recombinants is not detectable in natural isolates. Open Biol. 2018, 8, 170250. [CrossRef]

16. Seifert, R.; Schneider, E.H.; Bahre, H. From canonical to non-canonical cyclic nucleotides as second messengers: Pharmacological implications. Pharmacol. Ther. 2015, 148, 154-184. [CrossRef]

17. Belyy, A.; Raoux-Barbot, D.; Saveanu, C.; Namane, A.; Ogryzko, V.; Worpenberg, L.; David, V.; Henriot, V.; Fellous, S.; Merrifield, C.; et al. Actin activates Pseudomonas aeruginosa ExoY nucleotidyl cyclase toxin and ExoY-like effector domains from MARTX toxins. Nat. Commun. 2016, 7, 13582. [CrossRef]

18. Cowell, B.A.; Evans, D.J.; Fleiszig, S.M. Actin cytoskeleton disruption by ExoY and its effects on Pseudomonas aeruginosa invasion. FEMS Microbiol. Lett. 2005, 250, 71-76. [CrossRef]

19. Hritonenko, V.; Mun, J.J.; Tam, C.; Simon, N.C.; Barbieri, J.T.; Evans, D.J.; Fleiszig, S.M. Adenylate cyclase activity of Pseudomonas aeruginosa ExoY can mediate bleb-niche formation in epithelial cells and contributes to virulence. Microb. Pathog. 2011, 51, 305-312. [CrossRef]

20. Yahr, T.L.; Vallis, A.J.; Hancock, M.K.; Barbieri, J.T.; Frank, D.W. ExoY, an adenylate cyclase secreted by the Pseudomonas aeruginosa type III system. Proc. Natl. Acad. Sci. USA 1998, 95, 13899-13904. [CrossRef]

21. Morrow, K.A.; Ochoa, C.D.; Balczon, R.; Zhou, C.; Cauthen, L.; Alexeyev, M.; Schmalzer, K.M.; Frank, D.W.; Stevens, T. Pseudomonas aeruginosa exoenzymes $\mathrm{U}$ and $\mathrm{Y}$ induce a transmissible endothelial proteinopathy. Am. J. Physiol. Lung Cell. Mol. Physiol. 2016, 310, L337-L353. [CrossRef] [PubMed]

22. Balczon, R.; Pittet, J.F.; Wagener, B.M.; Moser, S.A.; Voth, S.; Vorhees, C.V.; Williams, M.T.; Bridges, J.P.; Alvarez, D.F.; Koloteva, A.; et al. Infection-induced endothelial amyloids impair memory. FASEB J. 2019, 33, 10300-10314. [CrossRef] [PubMed]

23. Lin, M.T.; Balczon, R.; Pittet, J.F.; Wagener, B.M.; Moser, S.A.; Morrow, K.A.; Voth, S.; Francis, C.M.; Leavesley, S.; Bell, J.; et al. Nosocomial Pneumonia Elicits an Endothelial Proteinopathy: Evidence for a Source of Neurotoxic Amyloids in Critically Ill Patients. Am. J. Respir. Crit. Care Med. 2018, 198, 1575-1578. [CrossRef] [PubMed]

24. Shaver, C.M.; Hauser, A.R. Relative contributions of Pseudomonas aeruginosa ExoU, ExoS, and ExoT to virulence in the lung. Infect. Immun. 2004, 72, 6969-6977. [CrossRef]

25. Schulert, G.S.; Feltman, H.; Rabin, S.D.; Martin, C.G.; Battle, S.E.; Rello, J.; Hauser, A.R. Secretion of the toxin ExoU is a marker for highly virulent Pseudomonas aeruginosa isolates obtained from patients with hospital-acquired pneumonia. J. Infect. Dis. 2003, 188, 1695-1706. [CrossRef]

26. Liu, P.V. The roles of various fractions of Pseudomonas aeruginosa in its pathogenesis. 3. Identity of the lethal toxins produced in vitro and in vivo. J. Infect. Dis. 1966, 116, 481-489. [CrossRef] 
27. Liu, P.V. The roles of various fractions of Pseudomonas aeruginosa in its pathogenesis. II. Effects of lecithinase and protease. J. Infect. Dis. 1966, 116, 112-116. [CrossRef]

28. Park, M.H.; Kim, S.Y.; Roh, E.Y.; Lee, H.S. Difference of Type 3 secretion system (T3SS) effector gene genotypes (exoU and exoS) and its implication to antibiotics resistances in isolates of Pseudomonas aeruginosa from chronic otitis media. Auris Nasus Larynx 2017, 44, 258-265. [CrossRef]

29. Pobiega, M.; Maciag, J.; Chmielarczyk, A.; Romaniszyn, D.; Pomorska-Wesolowska, M.; Ziolkowski, G.; Heczko, P.B.; Bulanda, M.; Wojkowska-Mach, J. Molecular characterization of carbapenem-resistant Pseudomonas aeruginosa strains isolated from patients with urinary tract infections in Southern Poland. Diagn. Microbiol. Infect. Dis. 2015, 83, 295-297. [CrossRef]

30. Pobiega, M.; Maciag, J.; Pomorska-Wesolowska, M.; Chmielarczyk, A.; Romaniszyn, D.; Ziolkowski, G.; Heczko, P.B.; Wojkowska-Mach, J.; Bulanda, M. Urinary tract infections caused by Pseudomonas aeruginosa among children in Southern Poland: Virulence factors and antibiotic resistance. J. Pediatr. Urol. 2016, 12, 36.e1-36.e6. [CrossRef]

31. Yousefi-Avarvand, A.; Khashei, R.; Sedigh Ebrahim-Saraie, H.; Emami, A.; Zomorodian, K.; Motamedifar, M. The Frequency of Exotoxin A and Exoenzymes S and U Genes Among Clinical Isolates of Pseudomonas aeruginosa in Shiraz, Iran. Int. J. Mol. Cell. Med. 2015, 4, 167-173.

32. Ochoa, C.D.; Alexeyev, M.; Pastukh, V.; Balczon, R.; Stevens, T. Pseudomonas aeruginosa exotoxin Y is a promiscuous cyclase that increases endothelial tau phosphorylation and permeability. J. Biol. Chem. 2012, 287, 25407-25418. [CrossRef] [PubMed]

33. Brummel, N.E.; Girard, T.D. Preventing delirium in the intensive care unit. Crit. Care Clin. 2013, $29,51-65$. [CrossRef] [PubMed]

34. Morris, A.C.; Hay, A.W.; Swann, D.G.; Everingham, K.; McCulloch, C.; McNulty, J.; Brooks, O.; Laurenson, I.F.; Cook, B.; Walsh, T.S. Reducing ventilator-associated pneumonia in intensive care: Impact of implementing a care bundle. Crit. Care Med. 2011, 39, 2218-2224. [CrossRef] [PubMed]

35. Pileggi, C.; Mascaro, V.; Bianco, A.; Nobile, C.G.A.; Pavia, M. Ventilator Bundle and Its Effects on Mortality Among ICU Patients: A Meta-Analysis. Crit. Care Med. 2018, 46, 1167-1174. [CrossRef]

36. Rivosecchi, R.M.; Kane-Gill, S.L.; Svec, S.; Campbell, S.; Smithburger, P.L. The implementation of a nonpharmacologic protocol to prevent intensive care delirium. J. Crit. Care 2016, 31, 206-211. [CrossRef]

37. Sen, S.; Johnston, C.; Greenhalgh, D.; Palmieri, T. Ventilator-Associated Pneumonia Prevention Bundle Significantly Reduces the Risk of Ventilator-Associated Pneumonia in Critically Ill Burn Patients. J. Burn Care Res. 2016, 37, 166-171. [CrossRef]

38. Ibanez, J.; Riera, M.; Amezaga, R.; Herrero, J.; Colomar, A.; Campillo-Artero, C.; De Ibarra, J.I.; Bonnin, O. Long-Term Mortality After Pneumonia in Cardiac Surgery Patients: A Propensity-Matched Analysis. J. Intensive Care Med. 2016, 31, 34-40. [CrossRef]

39. Karhu, J.; Ala-Kokko, T.I.; Ylipalosaari, P.; Ohtonen, P.; Laurila, J.J.; Syrjala, H. Hospital and long-term outcomes of ICU-treated severe community- and hospital-acquired, and ventilator-associated pneumonia patients. Acta Anaesthesiol. Scand. 2011, 55, 1254-1260. [CrossRef]

40. Wang, C.Y.; Calfee, C.S.; Paul, D.W.; Janz, D.R.; May, A.K.; Zhuo, H.; Bernard, G.R.; Matthay, M.A.; Ware, L.B.; Kangelaris, K.N. One-year mortality and predictors of death among hospital survivors of acute respiratory distress syndrome. Intensive Care Med. 2014, 40, 388-396. [CrossRef]

41. Balczon, R.; Morrow, K.A.; Zhou, C.; Edmonds, B.; Alexeyev, M.; Pittet, J.F.; Wagener, B.M.; Moser, S.A.; Leavesley, S.; Zha, X.; et al. Pseudomonas aeruginosa infection liberates transmissible, cytotoxic prion amyloids. FASEB J. 2017, 31, 2785-2796. [CrossRef]

42. Leber, A.L. (Ed.) Clinical Microbiology Procedures Handbook, 4th ed.; American Society for Microbiology: Washington, DC, USA, 2016.

43. Iglewski, B.H.; Sadoff, J.; Bjorn, M.J.; Maxwell, E.S. Pseudomonas aeruginosa exoenzyme S: An adenosine diphosphate ribosyltransferase distinct from toxin A. Proc. Natl. Acad. Sci. USA 1978, 75, 3211-3215. [CrossRef]

44. Anderson, D.M.; Sato, H.; Dirck, A.T.; Feix, J.B.; Frank, D.W. Ubiquitin activates patatin-like phospholipases from multiple bacterial species. J. Bacteriol. 2015, 197, 529-541. [CrossRef]

45. Benson, M.A.; Schmalzer, K.M.; Frank, D.W. A sensitive fluorescence-based assay for the detection of ExoU-mediated PLA(2) activity. Clin. Chim. Acta 2010, 411, 190-197. [CrossRef] 
46. Housley, N.A.; Winkler, H.H.; Audia, J.P. The Rickettsia prowazekii ExoU homologue possesses phospholipase A1 (PLA1), PLA2, and lyso-PLA2 activities and can function in the absence of any eukaryotic cofactors in vitro. J. Bacteriol. 2011, 193, 4634-4642. [CrossRef]

47. Jian, M.Y.; Alexeyev, M.F.; Wolkowicz, P.E.; Zmijewski, J.W.; Creighton, J.R. Metformin-stimulated AMPK-alpha1 promotes microvascular repair in acute lung injury. Am. J. Physiol. Lung Cell. Mol. Physiol. 2013, 305, L844-L855. [CrossRef]

48. Alvarez, D.F.; Housley, N.; Koloteva, A.; Zhou, C.; O'Donnell, K.; Audia, J.P. Caspase-1 Activation Protects Lung Endothelial Barrier Function during Infection-Induced Stress. Am. J. Respir. Cell. Mol. Biol. 2016, 55, 500-510. [CrossRef]

49. Khwaja, A. KDIGO clinical practice guidelines for acute kidney injury. Nephron Clin. Pract. 2012, 120, c179-c184. [CrossRef]

(C) 2020 by the authors. Licensee MDPI, Basel, Switzerland. This article is an open access article distributed under the terms and conditions of the Creative Commons Attribution (CC BY) license (http://creativecommons.org/licenses/by/4.0/). 